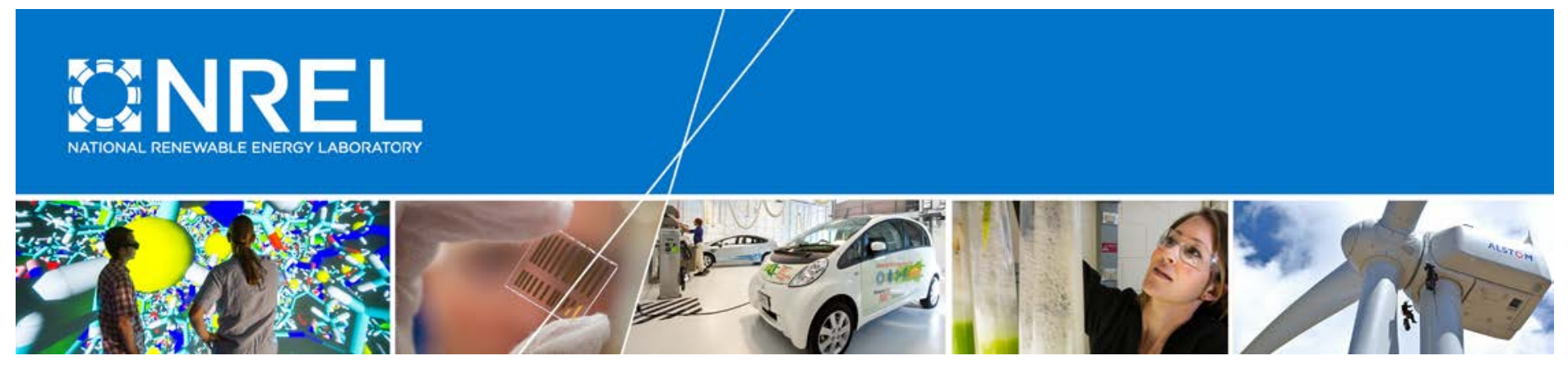

\title{
Opportunities and Challenges for Water and Wastewater Industries to Provide Exchangeable Services
}

Bethany Sparn and Randolph Hunsberger National Renewable Energy Laboratory

NREL is a national laboratory of the U.S. Department of Energy Office of Energy Efficiency \& Renewable Energy Operated by the Alliance for Sustainable Energy, LLC

This report is available at no cost from the National Renewable Energy Laboratory (NREL) at www.nrel.gov/publications.

Technical Report

NREL/TP-5500-63931

November 2015 


\section{Opportunities and Challenges for Water and Wastewater Industries to Provide Exchangeable Services}

Bethany Sparn and Randolph Hunsberger National Renewable Energy Laboratory

Prepared under Task No. BE4S.4445
NREL is a national laboratory of the U.S. Department of Energy Office of Energy Efficiency \& Renewable Energy Operated by the Alliance for Sustainable Energy, LLC

This report is available at no cost from the National Renewable Energy Laboratory (NREL) at www.nrel.gov/publications.

\section{Technical Report}

NREL/TP-5500-63931

November 2015

Contract No. DE-AC36-08G028308
National Renewable Energy Laboratory 15013 Denver West Parkway

303-275-3000 • www.nrel.gov 


\title{
NOTICE
}

This report was prepared as an account of work sponsored by an agency of the United States government. Neither the United States government nor any agency thereof, nor any of their employees, makes any warranty, express or implied, or assumes any legal liability or responsibility for the accuracy, completeness, or usefulness of any information, apparatus, product, or process disclosed, or represents that its use would not infringe privately owned rights. Reference herein to any specific commercial product, process, or service by trade name, trademark, manufacturer, or otherwise does not necessarily constitute or imply its endorsement, recommendation, or favoring by the United States government or any agency thereof. The views and opinions of authors expressed herein do not necessarily state or reflect those of the United States government or any agency thereof.

This report is available at no cost from the National Renewable Energy Laboratory (NREL) at www.nrel.gov/publications.

Available electronically at SciTech Connect http:/www.osti.gov/scitech

Available for a processing fee to U.S. Department of Energy and its contractors, in paper, from:

\author{
U.S. Department of Energy \\ Office of Scientific and Technical Information \\ P.O. Box 62 \\ Oak Ridge, TN 37831-0062 \\ OSTI http://www.osti.gov \\ Phone: 865.576.8401 \\ Fax: 865.576.5728 \\ Email: reports@osti.gov
}

Available for sale to the public, in paper, from:

\author{
U.S. Department of Commerce \\ National Technical Information Service \\ 5301 Shawnee Road \\ Alexandria, VA 22312 \\ NTIS http://www.ntis.gov \\ Phone: 800.553 .6847 or 703.605 .6000 \\ Fax: 703.605.6900 \\ Email: orders@ntis.gov
}




$\begin{array}{ll}\text { Acronyms } & \\ \text { EMWD } & \text { Eastern Municipal Water District } \\ \mathrm{kW} & \text { kilowatt } \\ \mathrm{kWh} & \text { kilowatt-hour } \\ \mathrm{MG} & \text { million gallons } \\ \text { MGD } & \text { million gallons per day } \\ \text { O\&M } & \text { operations and maintenance } \\ \text { SCADA } & \text { supervisory control and data acquisition } \\ \text { SCE } & \text { Southern California Edison } \\ \text { VFD } & \text { variable frequency drive } \\ \text { WTP } & \text { water treatment plant } \\ \text { WWTP } & \text { wastewater treatment plant }\end{array}$




\section{Executive Summary}

Water treatment plants (WTPs), wastewater treatment plants (WWTPs), and distribution systems use significant amounts of energy, about $2 \%-4 \%$ of the total electricity used in the United States; their energy use is projected to increase as populations increase and regulations become more stringent. Water and wastewater systems have largely been disconnected from the electric utilities' efforts to improve energy efficiency and provide grid services, likely because their core mission is to provide clean water and treated wastewater. Energy efficiency has slowly crept into the water and wastewater industry as the economic benefit has become more apparent, but there is still potential for significant improvement. Some of the larger, more progressive water utilities are starting to consider providing grid services; however, it remains a foreign concept to many. This report explores intrinsic mechanisms by which the water and wastewater industries can provide exchangeable services, the benefits to the parties involved, and the barriers to implementation. It also highlights relevant case studies and next steps. This report is intended to be a reference guide to the opportunities and challenges for WTPs and WWTPs to provide grid services.

Although process efficiencies can certainly be improved, this report focuses on the exchangeable services that water and wastewater loads can provide to help maintain grid reliability, keep overall costs down, and increase the penetration of distributed renewables on the electric grid. These services could provide water utilities additional value streams by using existing equipment with modest or negligible upgrade cost.

A few water and wastewater utilities have demonstrated the potential for their loads to be used for various grid services, and initial results have been promising. A number of flexible loads can be controlled in ways to assist the grid, ranging in scale from making frequent small adjustments to provide frequency regulation to turning off large loads for several hours for demand response. Most demonstrations have been carried out in WWTPs, even though WTPs or systems could also provide a number of grid services. The demonstrations have revealed a need for improved system design to accommodate additional storage or pump controls to add system flexibility if the water or wastewater utility wants to provide grid services for additional revenue. Water and wastewater utilities are less familiar with the needs of the electric grid, so encouraging more collaboration between water and wastewater utilities and electric utilities will help both types of utilities move their industries forward. 


\section{Table of Contents}

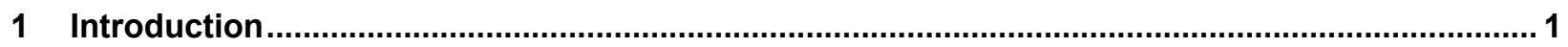

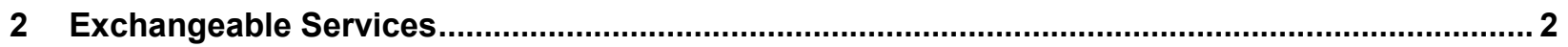

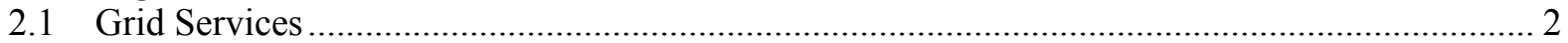

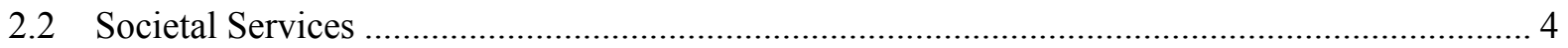

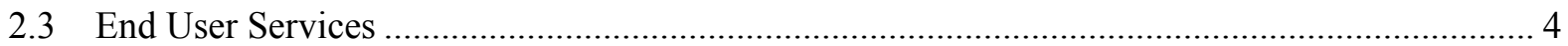

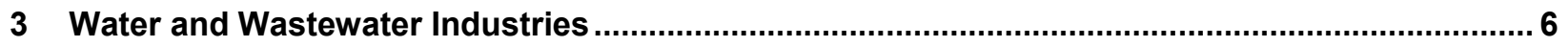

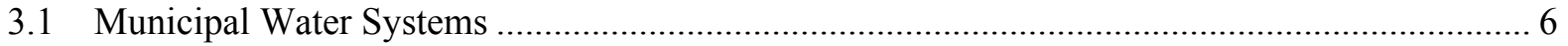

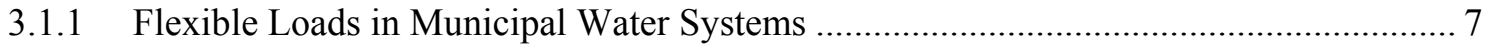

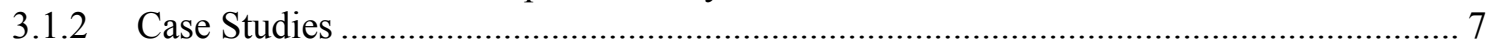

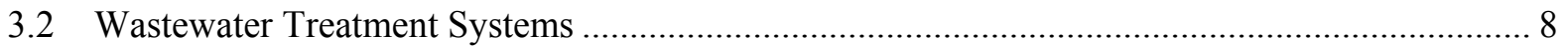

3.2.1 Flexible Loads in Wastewater Treatment Systems .................................................. 10

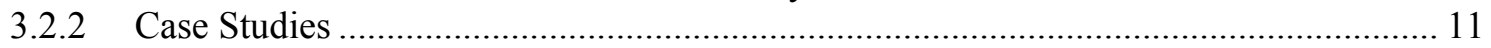

4 Conclusions

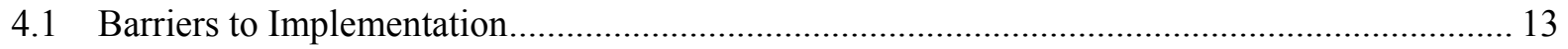

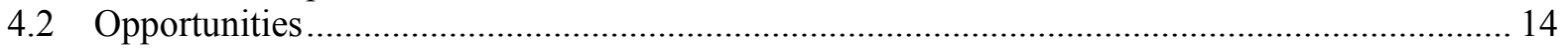

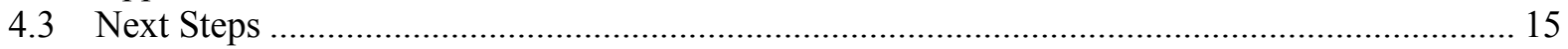

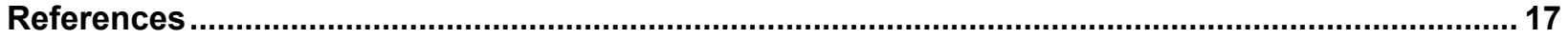




\section{List of Figures}

Figure 1. Generic water treatment process schematic. The equipment highlighted in yellow is of interest for providing grid services.

Figure 2. Generic WWTP process schematic. The equipment highlighted in yellow is of interest for

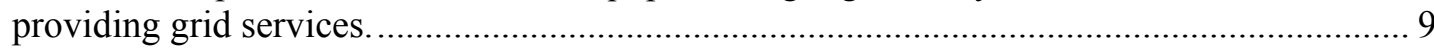




\section{Introduction}

Water treatment plants (WTPs), wastewater treatment plants (WWTPs), and distribution systems use significant amounts of energy, about $2 \%-4 \%$ of the total electricity used in the United States; their energy use is projected to increase as populations increase and regulations become more stringent (EPRI and WRF 2013; Liner and Stacklin 2013; Lekov et al. 2009). Water and wastewater systems have largely been disconnected from the electric utilities' efforts to improve energy efficiency and provide grid services, likely because their core mission is to provide clean water and treated wastewater. Energy efficiency has slowly crept into the water and wastewater industry as the economic benefit has become more apparent, but there is still potential for significant improvement. Some of the larger, more progressive water utilities are starting to consider providing grid services; however, it remains a foreign concept to many. This report explores intrinsic mechanisms by which the water and wastewater industry can provide exchangeable services, the benefit to the parties involved, and the barriers to implementation. It also highlights relevant case studies and next steps. This report is intended to be reference guide for the opportunities and challenges for WTPs and WWTPs to provide grid services.

Although process efficiencies can certainly be improved, this report focuses on the exchangeable services that water and wastewater loads can provide to help maintain grid reliability, keep overall costs down, and increase the penetration of distributed renewables on the electric grid. These services could provide water utilities additional value streams by using existing equipment with modest or negligible upgrade cost. 


\section{Exchangeable Services}

Exchangeable services are the valuable services and products that enable a transactional energy ecosystem and provide benefits to the parties participating in the transactions (Somasundaram et al. 2014). Exchangeable services can be in the form of an actual product, such as electric power, the delivery of information, or a financial product, such as an advanced purchase of energy (Somasundaram et al. 2014). Of the exchangeable services described by these authors, this report focuses on grid services, societal services, and end user services and how they relate to the water and wastewater industries.

Grid services include peak shifting and other ancillary services, along with improved planning and operations, which are used to support a reliable and flexible electric grid. Societal services provide benefits to society, such as enabling a larger percentage of renewable energy sources on the grid. End user services contribute to the overall controllability, efficiency, and predictability of the water and wastewater treatment infrastructure.

A fourth category of services - energy market services - also applies to the water and wastewater industries. These services are used to reflect the cost of producing and delivering electricity to end users, including flat rate structures and dynamic pricing structures such as time-of-use and real-time pricing. Water and wastewater utilities pay for their electricity according to the rate structure applied by their local electric utility, and some water utilities are subject to dynamic pricing. Although water and wastewater utilities could employ some interesting strategies to take advantage of variable rate structures, this report does not focus on this service, because it already applies to many water utilities. A number of the strategies for participating in grid services could easily be employed to reduce costs under a dynamic rate structure.

\subsection{Grid Services}

The electric grid has to constantly balance supply with demand while meeting power quality requirements (Ela, Milligan, and Kirby 2011). Demand fluctuates as countless individual loads turn on and off. Simultaneously, electricity generation, particularly from intermittent sources such as wind and solar power, ramps up and down. These two factors combine to cause voltage and frequency variations throughout the grid. Meeting overall power demands typically requires a mix of power generation: base generation such as nuclear and coal power (which cannot react quickly to changing demand) and faster-acting sources such as hydropower, gas turbines, diesel generators, and battery storage.

Grid services are used to ensure that the electricity supply is matched to demand. The categories of grid services include capacity, regulation, and contingency reserves, which are composed of spinning reserves, supplemental reserves, and replacement reserves. Capacity, also known as demand response, increases grid capacity in response to increased demand. Regulation and contingency reserves are often collectively referred to as ancillary services. These services are intended to maintain the reliability of the grid and are distinguished based on response speed, duration, and cycle time. For example, the purpose of regulation is to track rapid fluctuations in load. It is expected to react in less than a minute, operate for several minutes, and be able to cycle again in a few minutes. At the other end of the scale, replacement reserves are used to restore spinning and supplemental reserves, with a response speed of less than 30 minutes, a duration of up to 2 hours, with a cycle time of days. A final category is voltage control, which 
deals not with real power, but with reactive power. It is expected to be available continuously, with a response time measured in seconds. Not all these grid services are applicable to water and wastewater systems, but they are all described briefly for completeness.

Capacity provides "adequate resource availability," which can be achieved in a number of ways, including load control, generation control, and implementation of efficiency measures (Somasundaram et al. 2014). One distinction between capacity markets and the other grid services is that providing capacity is a promise for future action. A WTP or WWTP is paid to provide a specific quantity of capacity (kilowatts or megawatts), even if it is never asked to shed that load. Similarly, a gas turbine power plant can provide capacity by being available, even if it never needs to turn on. Capacity provides insurance to the electric utility that it will be able to provide enough power to meet demand at any time. Requirements for how quickly the load must be shed (or ramped up), duration, and frequency for calls are negotiated as terms for each contract.

Some ancillary services are needed during normal operation to compensate for constant fluctuations in demand. Regulation services are provided continuously to balance active power supply and demand and are automatically controlled by a centralized system (Ela, Milligan, and Kirby 2011). Load following (also known as flexibility) consists of additional resources that are used to compensate for differences between forecast and actual load; this is needed largely because of added renewable generation on the grid (Somasundaram et al. 2014; Olsen et al. 2013).

Other services are needed only in an emergency, such as a power plant or large load going offline. These are often referred to as contingency reserves (Ela, Milligan, and Kirby 2011). Frequency response (also known as spinning reserve or primary reserve) is used to automatically and instantaneously respond to a large active power imbalance, usually from the loss of a power plant. Supplemental or nonspinning reserves are also needed to compensate for the loss of generation during an emergency. Supplemental reserves take over for the frequency response reserves, and replacement reserves are used to take over for the supplemental reserves. The reserves that can respond fastest (frequency reserves) cannot maintain their response for long, whereas the reserves that can provide a longer response (supplemental and replacement reserves) cannot act as quickly. Many resources are used in an emergency to ensure that the grid can reliably provide quality power to all end users (Ela, Milligan, and Kirby 2011).

These descriptions are provided from the viewpoint of generation, but loads can also help to maintain the grid balance by ramping up or down as required. In some cases, using responsive loads (rather than generators) for grid balance has additional benefits, such as reducing the total current on a segment of a grid or managing utility infrastructure capital, operating, and contingency costs.

Some of these services can be provided through manual control, but those that require faster response typically need automated control, either with a signal provided by the electric utility, or by sensors that detect - and react to - frequency or voltage excursions beyond certain prescribed values. Open Automatic Demand Response (OpenADR) is an open Internet-protocol-based communication specification for sending demand response signals and currently appears to be the most common method used today for providing automatic control, especially in the case 
studies conducted in California (Thompson et al. 2010). Some companies, either control companies such as Honeywell or third-party aggregators such as Enbala, refer to their own branded communication systems. Whether these are built on OpenADR or are completely independent systems is unclear (Honeywell Building Solutions 2012; Enbala Power Networks 2012). Most modern WTPs and WWPTs have supervisory control and data acquisition (SCADA) systems to control various treatment processes, so each plant usually has a central control system that can be used to respond to automated grid services. The challenge arises when the water utility needs to understand how a certain action could affect more than a single plant, because system-wide data collection and control are not common at this point (WRF 2014).

\subsection{Societal Services}

In addition to the monetary benefit that water utilities would receive by participating in grid services, they would also enable societal services. Many of the grid services described in Section 2.1 are becoming more critical to the grid as more and more renewables are added to the generation mix (Ela, Milligan, and Kirby 2011). Rather than operating inefficient generators to stabilize the grid in the presence of large wind and solar sources, shedding or shifting loads would improve the overall carbon footprint of the grid. By providing ancillary services, water and wastewater utilities would be supporting higher penetrations of renewable energy, reducing carbon emissions, improving air quality, and enhancing system resiliency.

Providing valuable grid service to electric utilities could have additional societal benefits for water utilities. The added revenue earned by providing grid services could be used for capital improvements that increase system efficiency, which could then allow the water utilities to provide even more services. For instance, participating in capacity markets by turning loads on and off could pay for variable frequency drive (VFD)-driven pumps, which are more energy efficient and would allow the water utility to participate in regulation services. Indeed, some water utilities are finding that they can pay for significant upgrades by using energy more flexibly (Honeywell Building Solutions 2012).

\subsection{End User Services}

The last group of services that can be enabled by the transactional framework is end user services, which primarily benefit the operations and maintenance (O\&M) of the WTPs and WWTPs. The impact on O\&M is generally positive but needs more study in some cases. The use of VFDs with the many large pumps and motors used in water and wastewater systems has a number of O\&M benefits. VFDs allow pumps to be run at slower speeds when appropriate and offer soft-starting capabilities, which reduce the large in-rush current typically seen when a motor is turned on (EPRI and WRF 2013). The slower speeds and soft starts can reduce the wear and tear on pumps and motors, extending their lives and reducing maintenance costs. This also improves system reliability, because less downtime will be required for replacing pumps.

Another way O\&M can be improved is by implementing system-wide monitoring and control. A typical SCADA system at a single plant improves the operation of the plant, but few large water and wastewater utilities have the infrastructure in place to extend similar capabilities to their entire system. If that level of monitoring and control were needed to participate in various grid services, many opportunities would arise to improve operational control and reduce maintenance costs. The complex systems in water and wastewater utilities could benefit from a 
comprehensive control system that could collect information about electricity rate structures, demand response incentives, demands for water or wastewater treatment, maintenance needs, and weather trends. They could then act according to the priorities set by the operators.

Early efforts by the water and wastewater industries to participate in grid services (discussed in later sections) have been less sophisticated. In a few cases, capacity is provided by operators who manually turn pumps and motors off and on according to signals from the electric utilities. How these actions could impact the lifetime of the pumps and motors because of the increase in startups, especially for pumps without VFD control, is unclear. O\&M costs may not necessarily increase in these cases, but they must be considered. Additional research needs to be done to determine the overall impact that control strategies, including those that use VFDs, have on the maintenance costs and equipment lifetime. 


\section{Water and Wastewater Industries}

Water and wastewater treatment industries are largely driven by public health and environmental health. The U.S. Environmental Protection Agency and state health departments manage the rules that govern standards for clean drinking water and properly treated wastewater. These requirements are becoming increasingly more stringent. As such, the amount of energy required to deliver clean water is of lesser concern than ensuring that the clean water meets all the health requirements. Even as this mindset changes to include energy services the water and wastewater industries will not, above all else, do anything that could jeopardize their primary goal of providing clean water and treated wastewater.

\subsection{Municipal Water Systems}

In this report, the water system is defined as including the collection of raw water from surface or groundwater sources, treating it to meet regulatory standards, and delivering the potable water to customers. The energy associated with water end uses in homes and businesses is not considered here.

Generally, raw water comes from either surface water, such as reservoirs and rivers, or from groundwater via wells. A few treatment plants use desalination to treat water from the ocean, but that is rare because processing salt water is extremely energy intensive. Another minor source for water is recycled water from wastewater treatment. Between the plants that treat surface water and groundwater, the United States has more than 51,000 potable water treatment systems, most of which are very small. Eighty-two percent of the population is served with only $8 \%$ of the water treatment systems (EPRI and WRF 2013).

Daily water demand tends to look similar to daily electricity demand, with morning and evening peaks (EPRI and WRF 2013; Demand Response Research Center 2007). Peak water demand tends to occur during hot, dry weather, which also tends to produce peak electricity demands (EPRI and WRF 2013). Although the exact treatment system determines the energy intensity, larger capacity treatment plants tend to use less energy per volume of water treated than smaller plants. The smallest plants, which treat less than 3 million gallons per day (MGD), require about $2,000 \mathrm{kWh} / \mathrm{MG}$; the largest plants, which treat more than $20 \mathrm{MGD}$, use approximately 1,500 $\mathrm{kWh} / \mathrm{MG}$ (EPRI and WRF 2013). According to an analysis by EPRI and WRF, all community water treatment systems in the country used 38.6 terawatt-hours (0.13 Quads) for the 2011 calendar year (EPRI and WRF 2013).

Treatment processes vary, but pumping accounts for most of the electricity used in water treatment systems, about $55 \%-90 \%$ of the total load depending on the details of hydraulic system and treatment process (EPRI and WRF 2013; California Sustainability Alliance 2012). As previously mentioned, desalination is very energy intensive and uses up to 10 times more energy than a more conventional method, so the fraction of energy use from pumping is very different for desalination plants (EPRI and WRF 2013).

Three pumping systems — raw water pumping, in-plant pumping, and finished water pumpingare at work in a water treatment system. The raw water pumping is needed to deliver the raw water to the treatment plant, where it is then pumped through all the processes, and then the finished water must be delivered to end users at a certain pressure (see Figure 1). In-plant 
pumping is not shown in Figure 1, but it would be used to move water through the treatment steps of coagulation/flocculation, sedimentation, filtration, and disinfection. Finished water pumping - all the pumping needed to take the water from the treatment plant to customers - is the largest load. Distribution system pressure is sometimes achieved by pumping to an elevated tank and then using gravity to supply end users. Generally, a community water system has either an elevated storage tank or ground level storage, but not both. To deliver the water to the end users, pumping stations throughout a city are used to maintain mains water pressure.

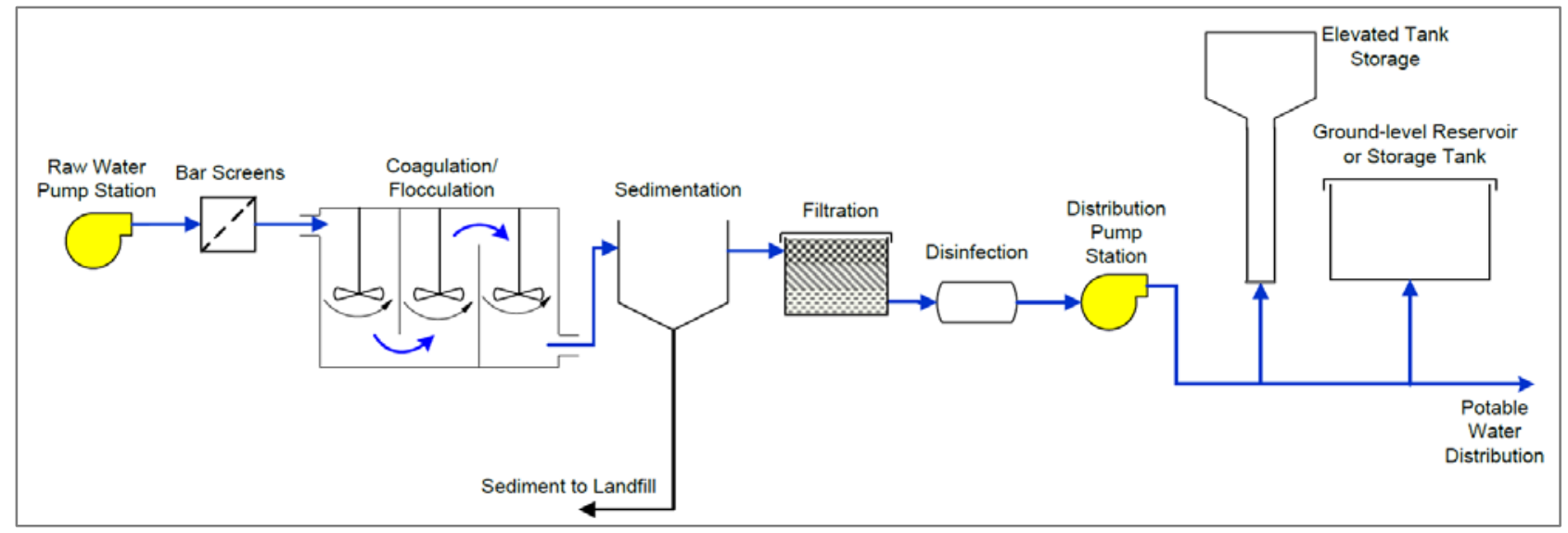

Figure 1. Generic water treatment process schematic. The equipment highlighted in yellow is of interest for providing grid services.

\subsubsection{Flexible Loads in Municipal Water Systems}

The various pumps throughout the water system have different degrees of flexibility, depending on the system configuration. Some arid localities such as California and Colorado draw their raw water from distant sources through a series of reservoirs and storage tanks (EPRI and WRF 2013). Those systems have a high level of flexibility in raw water pumping that can be controlled to either provide fast grid services such as regulation or longer services such as capacity. If the raw water comes from a nearby reservoir or river and does not have to travel far, raw water pumping may have less flexibility.

Finished water pumping includes pumping potable water to tanks or reservoirs and the pumping needed to pressurize mains water lines and deliver water to end users. The pumping energy required to deliver water to storage tanks is flexible, and many water utilities already wait to pump to storage tanks at night when electricity rates are lower (Raucher et al. 2008). Potable water could be pumped to storage tanks to provide regulation services or capacity services. Because many localities already pump at night, the ability to provide capacity services would depend on which strategy gives the water utility the most benefit.

In-plant pumping energy use might be reduced by using VFDs and running at slower speeds, but this has not been explored. Additionally, in-plant pumping is the smallest of the three pumping loads and so would provide the smallest benefit (EPRI and WRF 2013).

\subsubsection{Case Studies}

Few demonstrations have used water treatment and distribution loads for grid services, and fewer still have been reported in detail. Most case studies that involve municipal water systems do not 
offer specific details about the loads that are being controlled. Pumping is the most likely candidate, but few details are available about which pumping loads are being controlled. A few case studies are summarized in the following sections.

\subsubsection{Eastern Municipal Water District}

Eastern Municipal Water District (EMWD) in California has enrolled in Southern California Edison's (SCE's) EnerNOC Demand Response Program. EnerNOC is a third-party aggregator that facilitates the connection between EMWD and SCE. When a demand response event occurs, pumps at two filtration plants are manually shut down by operators at EMWD and can remain off for a couple hours without impacting overall water delivery. For its participation, EMWD is paid $\$ 100,000$ annually (EnerNOC 2008). EMWD is also directly enrolled in a Base Interruptible Program and Price-Based programs for day-ahead pricing with SCE. The combined savings for participation in these three grid and energy market programs is $\$ 555,000$ annually for EMWD (California Sustainability Alliance 2012). EMWD also plans to partner with Honeywell to convert some of its manual controls to automatic controls to increase its demand response portfolio.

\subsubsection{Cucamonga Valley Water District}

The Cucamonga Valley Water District in California, with help from Honeywell, participates in SCE's Automated Demand Response program (Honeywell Building Solutions 2012). This district includes water and wastewater treatment and provides 5.3 megawatts of capacity for up to 2 hours, which represents $40 \%$ of its total load. Control is automatic (Akuacom by Honeywell Demand Response Management System software was used). The program was expected to provide more than $\$ 400,000$ per year in revenue for the utility, but no updates have been made to verify the accuracy of this projection. At the very least, the initial rebate was sufficient to pay for the equipment upgrades that enabled the district to participate in the Automated Demand Response program and will help the utility with system-wide control and efficiency.

\subsubsection{Hawaiian Electric Companies}

The Hawaiian Electric Companies plan to control water and wastewater pumping loads to provide up to 2.4 megawatts of regulation reserve, but how much of that total potential comes from municipal water pumping is unclear (Hawaiian Electric Company 2014). They have not yet begun this program and are looking for information from private companies to help them promote demand response and collaboration with the water and wastewater utilities (Rosegg 2014).

\subsection{Wastewater Treatment Systems}

Wastewater treatment systems, for this discussion, are defined as all processes needed to collect wastewater in the service area, send it through a treatment plant, and discharge the treated water and solids to their proper locations (Figure 2). The discussion is limited to central treatment plants and excludes septic systems that may be common in rural areas. 


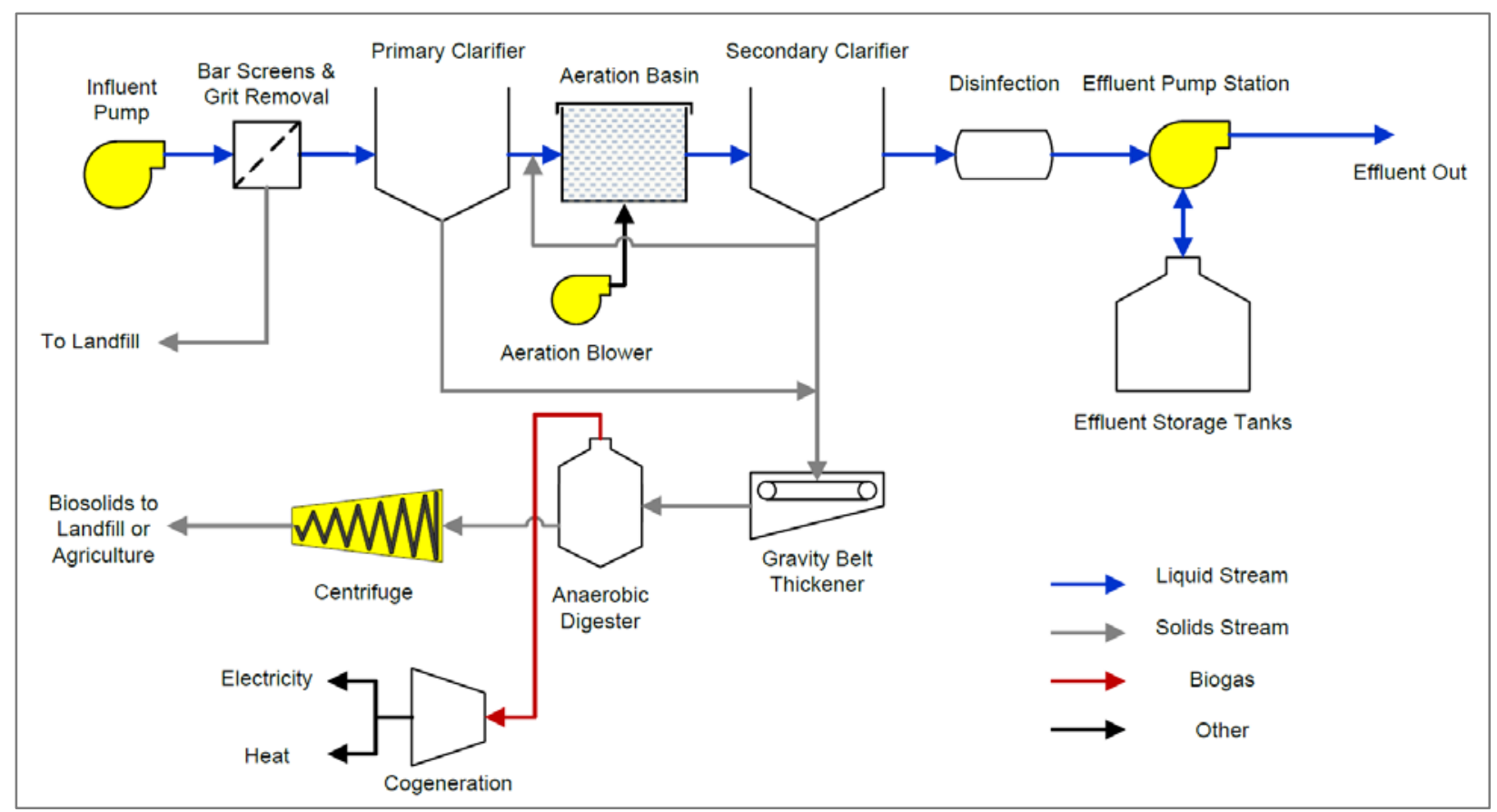

Figure 2. Generic WWTP process schematic. The equipment highlighted in yellow is of interest for providing grid services.

Adapted from Olsen et al. (2012)

The United States has fewer than 15,000 distinct WWTPs, serving about $75 \%$ of the population (EPRI and WRF 2013). The other quarter of the population is served by septic and other on-site systems. As with water treatment systems, most wastewater treatment systems are small; $90 \%$ of the treatment capacity is provided by $20 \%$ of the treatment systems.

The flow of wastewater into a WWTP generally has a daily profile that is similar to the potable water use profile, but it is delayed slightly. Peaks in influent flow in the morning and evening occur after the demand peaks at a WTP (Thompson et al. 2010). As with WTPs, smaller plants have higher energy intensity per volume of influent treated than larger plants. The smallest WWTPs, treating less than $2 \mathrm{MGD}$, require about 3,300 $\mathrm{kWh} / \mathrm{MG}$. The largest treatment plants - larger than $100 \mathrm{MGD}$ - use about 1,600 kWh/MG (EPRI and WRF 2013). The Electric Power Research Institute and Water Research Foundation estimate that all the U.S. municipal WWTPs used 30.2 terawatt-hours (0.10 Quads) in 2011 (EPRI and WRF 2013).

Most energy used in wastewater treatment systems is in the treatment processes, unlike water treatment systems in which the actual treatment processes use only about $15 \%$ of the total energy consumed. The largest load in wastewater treatment comes from aeration blowers, often about $50 \%$ of the total load, in contrast to pumping, which contributes about $15 \%$ of the load for wastewater treatment (EPRI and WRF 2013; Thompson et al. 2008). Wastewater can be adequately treated in many ways; the size of the treatment facility plays an important role in the treatment system chosen. Regulations that dictate the content of the final products, the effluent, and the solid waste are regularly updated to be more restrictive, which also impacts the treatment methods used. 
Another factor in wastewater treatment is whether the sanitary sewer is separate from or combined with the storm sewer. An older city is more likely to have a combined sewer system, which means that the volume of water to be treated rises dramatically after a storm (Olsen et al. 2012). Cities with combined sewers tend to have more storage built into their collection systems (upstream of the WWTP) and to have facilities that can handle much larger volumes of influent than their average load. Despite the many reasons that combined sewer systems should be avoided, the resulting system has a lot of built-in buffer that could provide an opportunity to participate in various grid services (EPRI and WRF 2013).

\subsubsection{Flexible Loads in Wastewater Treatment Systems}

Various loads, including the effluent pumps, centrifuges for dewatering sludge, blowers for aeration, and even the entire plant can be controlled in a WWTP. WWTPs may also be able to reduce their demand from the grid by turning on generators powered by biogas that is produced onsite. This is especially appealing if the site cannot produce enough biogas to run a generator continuously or if temporarily shutting down certain processes can cause problems.

Fast load following services such as frequency regulation might be accomplished with a VFDdriven pump ramping up and down to follow the market signal without deviating far from normal operation. This would require the process being driven by the pump to be moderately flexible. The pump would still be running but may not be able to hit very precise set points such as system pressure. Deviations from the most efficient operating point of the pump would also cause an overall decrease in energy efficiency, but revenue gained by providing regulation service could more than offset the efficiency losses (Enbala Power Networks 2012).

Slower services such as capacity would require a load that could be altered significantly for a longer period of time. For instance, if the collection system has sufficient storage, an entire plant could be turned off for periods of time - on the order of hours - without adversely affecting the long-term performance of the system. Other ways to reduce loads for finite periods include holding effluent in a local storage tank to avoid using the effluent pumps and delaying use of a centrifuge by collecting solid waste in a holding tank.

One case study looked at turning off aeration blowers for several hours, but that resulted in a spike in turbidity after normal operation resumed (Thompson et al. 2010). The rise in turbidity is an unacceptable side effect of a demand response event, but the load for variable- and multispeed blowers might be reduced without adversely affecting overall operations. Another option to reduce the aeration load during peak times is to overoxygenate the aeration tank before a capacity event to ensure that the dissolved oxygen levels do not fall too much (Lekov et al. 2009; EPRI and WRF 2013).

On-site electricity generation with biogas would be best suited to longer services such as capacity. When a reduction in load is called for, biogas generators could power some large loads to reduce the grid demand without impacting the plant operation. This is a viable control strategy only if the biogas generators are not already running when the service is called for. Many generators run constantly at WWTPs and so would not be available to provide grid services (Olsen et al. 2012). Generators are generally run constantly if enough biogas is produced onsite to support constant operation. Electricity contracts may also factor into the ability for biogas generators to provide grid services. In at least one case, a WWTP using biogas for electricity 
production in southern California was required to always be a net consumer of electricity from its local utility, which limited its ability to participate in grid services (Thompson et al. 2010).

Capacity is the most common service currently provided by WWTPs. Plants that participate in capacity markets curtail 5\%-40\% of the total plant load during an event (Rulseh 2012; Olsen et al. 2013). Generally, power consumption at WWTPs is fairly flat throughout the day and year (Olsen et al. 2013). If we take the 2011 annual energy consumption estimate from the Electric Power Research Institute and Water Research Foundation and assume that power consumption is constant throughout the year, aggregate power consumption for all wastewater treatment systems is about 3.4 gigawatts. Based on the most conservative estimate of $5 \%$ of total plant load being available for capacity markets, the wastewater treatment industry could provide 170 megawatts of capacity to the grid. This is a rough and very conservative estimate, but it does give some sense of the potential scale for using wastewater treatment loads for capacity markets.

\subsubsection{Case Studies}

In contrast to community water systems, several case studies have looked at ways that loads in WWTPs could be used for grid services. California utilities have been actively exploring ways that WWTPs could be used for grid services, and a couple of third-party aggregators have employed some innovative strategies. A WWTP has more options for control, because it has a number of energy-intensive end loads. This may explain why more demonstrations have been conducted in wastewater systems relative to municipal water systems. Three case studies are summarized in the following sections.

\subsubsection{Pennsylvania American Water}

Pennsylvania American Water, a subsidiary of American Water, partnered with Enbala Power Networks to provide frequency regulation to the Pennsylvania-New Jersey-Maryland market (Enbala Power Networks 2012; EPRI and WRF 2013). For the pilot, a single 700-horsepower pump at a pumping station was connected to Enbala's GOFlex platform, which automatically ramped the pump up and down within a narrow range to provide frequency regulation. In return for this constant participation, Pennsylvania American Water is paid \$20,000 annually or 2\%$3 \%$ of the pumping station's annual energy bill (Enbala Power Networks 2012). Operators at the pump station see no noticeable difference in service or operation and American Water plans to enroll other assets in the Pennsylvania-New Jersey-Maryland frequency regulation market. In this case, no functionality in the plant was lost or delayed. The pump was constrained to operate between $85 \%$ and $100 \%$ but was never turned off.

\subsubsection{City of Oceanside}

The City of Oceanside in southern California partnered with Lawrence Berkeley National Laboratory to determine if one of its municipal WWTPs, the San Luis Rey facility, could provide demand response. Effluent pumps, centrifuges, and aeration blowers were submetered to evaluate potential. Manual demand response testing was then conducted (Thompson et al. 2010). Generally, the results were positive because the pumps and centrifuges were good candidates for demand response. However, turning off the aeration blowers for 2 hours resulted in a spike in turbidity a few hours after normal operation resumed. This result was unacceptable for the plant operators and they will not consider control of the aeration blowers in the future. The pumps and centrifuges together could provide about $215 \mathrm{~kW}$ of peak load reduction. However, the San Luis 
Rey facility has a 600-kW cogeneration plant and San Diego Gas \& Electric requires the WWTP plant to be a net consumer at all times. This requirement could restrict how much load reduction it could provide for demand response.

\subsubsection{Southeast Water Pollution Control Plant}

As a follow-on to the San Luis Rey facility demonstration, Lawrence Berkeley National Laboratory worked with the Southeast Water Pollution Control Plant in San Francisco to determine if the demand response potential found in Oceanside would be applicable at a larger facility (Olsen et al. 2012). The Southeast plant treats water from a combined sewer and storm water system in San Francisco, so it experiences large swings in influent volume between winter (the wet season) and summer (the dry season). Based on the results from the San Luis Rey plant, the aeration blowers were not considered for this demonstration. Four influent lift pumps and six centrifuges were submetered and subjected to short-term testing to determine their demand response potential, which was $240 \mathrm{~kW}$. In addition to conducting deliberate tests of the pumps and centrifuges, researchers were also able to use maintenance events to evaluate some other measures that the plant operators were reluctant to test for demand response. They found that a partial plant shutdown was the most effective, providing nearly $1,000 \mathrm{~kW}$ of capacity at the plant and another $1,000 \mathrm{~kW}$ from the pump stations that fed the plant. Although a plant shutdown is not always feasible, it may be possible for 5-10 hours as long as the weather is dry, because the combined sewer/storm water system provides several days' worth of influent storage. 


\section{Conclusions}

\subsection{Barriers to Implementation}

The research done to date suggests that WTPs and WWTPs could be excellent partners for their electric utility counterparts, because they can provide a variety of grid services. However, some case studies cite the lack of relationship between the electric and water utilities as a significant barrier to progress (WRF 2014; Liner and Stacklin 2013). Water utilities focus entirely on their main business of providing clean drinking water and treating wastewater for disposal. Grid services are not a well-known concept in the water industry, so electric utilities need to reach out to their water utility counterparts to start the conversation (SCE 2008).

Also, governmental and academic assessments of the potential for new industries to provide grid services appear to be inconsistent. A number of studies have evaluated the resources in the industrial sector to provide grid services; some include wastewater treatment (but not water treatment) and some do not include the water industry (Goli et al. 2011; Starke, Alkadi, and Ma 2013). This is also true of studies looking at the water-energy nexus. A joint analysis by the Alliance for Water Efficiency and the American Council for an Energy-Efficient Economy identified the lack of "estimates of technical and economic energy efficiency and demand response potential in water and wastewater systems and industry accepted guidelines for doing such studies on individual systems" as a major research gap (GEI Consultants, Inc. 2013). However, a recent U.S. Department of Energy analysis of the water-energy nexus mentioned (but did not emphasize) demand response in the context of WWTPs only (DOE 2014). The water industry is not seen as a natural partner for the electricity industry — even by those who evaluate the connection between the two industries - so changing this perception will require some effort.

Financial incentives are generally the main motivation for water utilities to provide grid services, so those incentives need to be large enough to justify the added complication and potential impact on efficiency and plant performance. In 2008, SCE offered $\$ 17 / \mathrm{kW}$ to the utilities for participating in its demand response program, which was deemed too low for many WWTP managers (SCE 2008). In 2012, Randy Palombi with Constellation NewEnergy, a third-party aggregator, estimated the nationwide average to be $\$ 60 / \mathrm{kW}$ for capacity services, so rates might have been raised to encourage participation (Rulseh 2012). Whether lack of incentives is a barrier is unclear at this point, but it remains an important factor for water and wastewater utilities that are considering providing grid services.

Financial incentives could be structured in creative ways to help water utilities install the controls and equipment they need to participate in certain services in return for a long-term commitment to provide grid services. Upgrades needed to provide grid services often lead to overall system efficiency improvements and control systems with increased reliability.

In addition to ensuring attractive financial incentives, electric utility policies need to be reviewed to ensure that water utilities are not penalized for certain aspects of their participation. Some utilities charge penalties if customers providing capacity cannot participate in a single event (SCE 2008). Especially in industries such as water and wastewater that focus on public health, individual events need to be accommodated. Also, in one case study in Port Angeles, Washington, a customer was asked to participate in a load-add event but declined because the monthly demand charge would have increased as a result (EPRI and WRF 2013). Providing grid 
services to the electric utility should be mutually beneficial, so the rules and penalties need to be evaluated to ensure that all parties receive the anticipated benefit.

Even though some estimates show that water treatment and distribution systems use slightly more energy than wastewater treatment systems across the country, water systems are rarely considered for analysis or demonstrations of grid services. The origin of this bias is unclear, because water and wastewater treatment systems seem equally well positioned to provide grid services. Pumping loads, which are easily controlled with VFDs, make up the bulk of the water system load, and storage is always built in to the distribution network. The United States has many more distinct water treatment facilities than wastewater treatment facilities $(51,000$ WTPs versus 15,000 WWTPs), so targeting WWTPs might be more time and cost effective. Regardless of the reason, water treatment and distribution system resources present a largely untapped opportunity.

\subsection{Opportunities}

The water and wastewater system infrastructure presents a number of opportunities that would enable it to provide more grid services. A key finding from the research is that system design of WTPs and WWTPs could be used to make their processes more flexible and more responsive to grid needs without impacting their core mission. Building more storage throughout the system, properly locating the storage, installing VFD-driven pumps when appropriate, and putting pumps in series would all enable facilities to better respond to the grid. Working with the local electric utility when designing new treatment plants or when planning for upgrades may open more opportunities to provide grid services and generate extra revenue (WRF 2014). Along the same lines, water and wastewater utilities need tools to evaluate the state of their entire infrastructure. A single plant may have a central SCADA system that can be used to monitor and control the entire plant, but water utilities do not necessarily have an equivalent tool to monitor and control all their treatment plants, storage tanks, and distributed pump stations (WRF 2014). Achieving the system-wide flexibility that demand response participation requires will be difficult without system-wide management.

Biogas can provide a renewable source of power to WWTPs and can be stored for use during a demand response event to ease the impact on the plant. However, hundreds of plants - for a number of reasons - produce biogas and do not use it to make electricity (Liner and Stacklin 2013). To use biogas to generate electricity, it must be cleaned more than is needed to just flare the biogas, and the additional infrastructure is expensive (EPRI and WRF 2013). In addition to the extra processing and cost, regulatory hurdles may make the use of biogas for electricity even less appealing. Air quality emissions limits in southern California may force a number of biogas generators to shut down operation (California Sustainability Alliance 2012). In some instances the plant is contractually obligated by the electric utility to always be a net consumer of energy. That may prevent some plants from using biogas for supplemental electric power during demand response events, even though that strategy would alleviate many of the concerns associated with shutting off equipment for short periods in WWTPs. Especially in plants that are already producing biogas but not using it for electricity, assistance could be given to encourage the use of biogas for electricity for grid services. Electric utilities and WWTPs may also find opportunities to work together to allow the plant to occasionally be a net producer of electricity when the electric grid needs additional generation. 
Some case studies included a third-party aggregator who helped to bridge the gap between water and electric utilities. A water utility may receive slightly reduced revenue by working with a third-party aggregator to provide grid services, but the operators also would not need to become experts in grid services and train staff to act on grid service requests. An additional benefit cited by EMWD is that electric utilities often charge penalties to their grid service providers if they opt out of an event, whereas the aggregators do not charge a penalty (Atkinson 2013). The WTP or WWTP would not be paid for an event that it opted out of, but it would not be penalized further.

\subsection{Next Steps}

- Even though a handful of demonstration projects have looked into the viability of using water and wastewater systems for demand response, more work still needs to be done. According to the Alliance for Water Efficiency and the American Council for an EnergyEfficient Economy water-energy nexus report, key questions remain: "What percentage of a water or wastewater facility energy load can reasonably be shifted or reduced? What service or activity is most affected by demand response?" (GEI Consultants, Inc. 2013). More demonstrations evaluating exchangeable services should be implemented in the water and wastewater sectors to answer the questions that still remain. Learning more about how water and wastewater treatment systems can participate in grid services will help lessen the risk to others that want to take advantage of this additional revenue stream.

- Technical factors related to cyclical operations of pumps, variable-speed pumps, and variable-speed drives need to be evaluated to determine possible impacts on system operation, reliability, maintenance requirements, and component life (Kirby 2003).

- Most of the research to date has been conducted at WWTPs. Even though a few demonstrations have been done with municipal water systems, the published results contain few details that will help other WTPs that are considering participation in grid services. As such, detailed and rigorous demonstration projects with water treatment and distribution utilities are needed. Every water system is different, so evaluating the potential at large and small WTPs would be valuable. Many questions remain about the best ways to implement grid services and the potential impacts on the rest of the system.

- Only one case study about a water or wastewater utility providing ancillary services has been published. Although it was a successful demonstration, few details are available to the public (Enbala Power Networks 2012). Because some ancillary services have the potential to provide revenue with less impact on the system than capacity services, they may be more attractive to water and wastewater utilities. However, more research needs to be done to determine which loads are the best fit and to ensure that the services can be provided without unintended consequences.

- Water and energy are interdependent. As water becomes scarcer and energy is provided by more renewable sources, the need for the electric and water utility sectors to work together becomes more urgent. Using water and wastewater infrastructure to provide grid services is just one way that water and electric utilities could work together. As a followon to the Water Energy Nexus Report from 2014, the U.S. Department of Energy could facilitate workshops for electric utilities and water and wastewater utilities to start a dialogue between the different industries. The electric utilities need partners to expand 
participation in various grid services, and water utilities are looking for ways to increase revenue without increasing rates. Bringing these utilities together would allow electric utilities to explain their needs for demand response and ancillary services and what those might look like in a WTP or WWTP. Water and wastewater utilities would be given the opportunity to describe their distribution and treatment systems, which loads are most critical, and which loads have inherent flexibility. The open dialogue may help to identify new opportunities and partnerships. 


\section{References}

Atkinson, W. 2013. "Balance of Power: Water Utilities Find Ways To Reduce Energy Costs through Demand Response." WaterWorld. October 1, 2013. Accessed February 12, 2015: www.waterworld.com/articles/print/volume-29/issue-10/editorial-features/balance-ofpower-water-utilities-find-ways-to-reduce-energy-costs-through-demand-response.html.

California Sustainability Alliance. 2012. Eastern Municipal Water District: A Case Study of Best-In-Class Water-Energy Programs and Practices. San Francisco, CA: California Sustainability Alliance.

Demand Response Research Center. 2007. Water Suppy-Related Electricity Demand in California. Sacramento, CA: California Energy Commission.

DOE. 2014. The Water-Energy Nexus: Challenges and Opportunities. Washington, D.C.: U.S. Department of Energy.

Ela, E., M. Milligan, and B. Kirby. 2011. Operating Reserves and Variable Generation (Technical Report, NREL/TP-5500-51978). Golden, CO: National Renewable Energy Laboratory.

EPRI, and WRF. 2013. Electricity Use and Management in the Municipal Water Supply and Wastewater Industries. Palo Alto, CA: Electric Power Research Institute and Denver, CO: Water Research Foundation.

Enbala Power Networks. 2012. Pennsylvania American Water Connects to the Smart Grid. Toronto, OR: Enbala Power Networks Inc.

EnerNOC. 2008. Eastern Municipal Water District Works with EnerNOC To Reduce Significant Electrical Load. Boston, MA: EnerNOC.

GEI Consultants, Inc. 2013. Water-Energy Nexus Research: Recommendations for Future Opportunities. Alliance for Water Efficiency; American Council for an Energy Efficient Economy, Project No. 130240.

Goli, S., D. Olsen, A. McKane, and M.A. Piette. 2011. 2008-2010 Research Summary: Analysis of Demand Response Opportunities in California Industry (Technical Report, LBNL-5680E). Berkeley, CA: Lawrence Berkeley National Laboratory.

Hawaiian Electric Company. 2014. Integrated Demand Resource Portfolio Plan. Honolulu, HI: Hawaii Public Utilities Commission.

Honeywell Building Solutions. 2012. Honeywell Helps Water District Shed 40 Percent of Load; Achieve Savings. Golden Valley, MN: Honeywell.

Kirby, B. 2003. Spinning Reserve from Pump Load: A Technical Findings Report to the California Department of Water Resources (Technical Report, ORNL/TM-2003/99). Oak Ridge, TN: Oak Ridge National Laboratory. 
Lekov, A., L. Thompson, A. McKane, K. Song, and M.A. Piette. 2009. Opportunities for Energy Efficiency and Open Automated Demand Response in Wastewater Treatment Facilities in California - Phase I Report (Technical Report, LBNL-2572E). Berkeley, CA: Lawrence Berkeley National Laboratory.

Liner, B., and C. Stacklin. 2013. "Driving Water and Wastewater Utilities to More Sustainable Energy Management.” Proceedings of the ASME 2013 Power Conference. Boston, MA.

Olsen, D., N. Matson, M.D. Sohn, C. Rose, J. Dudley, S. Goli, and S. Kiliccote. 2013. Grid Integration of Aggregated Demand Response, Part I: Load Availability Profiles and Constraints for the Western Interconnection (Technical Report), LBNL-6417E). Berkeley, CA: Lawrence Berkeley National Laboratory.

Olsen, D., S. Goli, D. Faulkner, and A. McKane. 2012. Opportunities for Automated Demand Response in Wastewater Treatment Facilities in California - Southeast Water Pollution Control Plant Case Study (Technical Report, LBNL-6056E). Berkeley, CA: Lawrence Berkeley National Laboratory.

Raucher, R.S., J.E. Cromwell, K. Cooney, P. Thompson, L. Sullivan, B. Carrico, M. MacPhee, and R. Wilkinson. 2008. Risks and Benefits of Energy Management for Drinking Water Utilities. Denver, CO: AWWA Research Foundation.

Rosegg, P. 2014. "Hawaiian Electric Seeks Information on Private Partners To Boost Electricity Demand Management." December 2, 2014. Accessed February 13, 2015: www.hawaiianelectric.com/heco/ hidden Hidden/CorpComm/Hawaiian-Electric-seeksinformation-on-private-partners-to-boost-electricity-demand-management?cpsextcurrchannel=1.

Rulseh, T. 2012. "Power Down, Revenue Up." Treatment Plant Operator. May 2012. Accessed February 12, 2015: www.tpomag.com/editorial/2012/05/power_down_revenue_up.

Somasundaram, S., R.G. Pratt, B.A. Akyol, N. Fernandez, N. Foster, S. Katipamula, E.T. Mayhorn, A. Somani, A. Steckley, and Z.T. Taylor. 2014. Reference Guide for a TransactionBased Building Controls Framework (Technical Report, PNNL-23302). Richland, WA: Pacific Northwest National Laboratory.

SCE.2008. Demand Response Feasibility Study for the Sewage Treatment Industry. Rosemead, CA: Southern California Edison, DR 08.03 Report.

Starke, M., N. Alkadi, and O. Ma. 2013. Assessment of Industrial Load for Demand Response across U.S. Regions of the Western Interconnect (Technical Report, ORNL/TM-2013/407). Oak Ridge, TN: Oak Ridge National Laboratory.

Thompson, L., A. Lekov, A. McKane, and M.A. Piette. 2010. Opportunities for Automated Demand Response in Wastewater Treatment Facilities in California - Phase II Report: San Luis Rey Wastewater Treatment Plant Case Study (Technical Report, LBNL-3889E). Berkeley, CA: Lawrence Berkeley National Laboratory. 
Thompson, L., K. Song, A. Lekov, and A. McKane. 2008. Automated Demand Response Opportunities in Wastewater Treatment Facilities (Technical Report, LBNL-1244E). Berkeley, CA: Lawrence Berkeley National Laboratory.

WRF. 2014. Trends, Challenges and Opportunities for Energy Management in Water and Wastewater Utilities. Denver, CO: Water Research Foundation, \#4356. 